\title{
RNA Editing Genes Associated with Extreme Old Age in Humans and with Lifespan in C. elegans
}

\author{
Paola Sebastiani ${ }^{19 *}$, Monty Montano ${ }^{29 *}$, Annibale Puca ${ }^{3}$, Nadia Solovieff ${ }^{1}$, Toshio Kojima ${ }^{4}$, Meng C. \\ Wang $^{5}$, Efthymia Melista ${ }^{6}$, Micah Meltzer ${ }^{2}$, Sylvia E. J. Fischer ${ }^{5}$, Stacy Andersen ${ }^{7}$, Stephen H. Hartley ${ }^{1}$, \\ Amanda Sedgewick ${ }^{8}$, Yasumichi Arai ${ }^{9}$, Aviv Bergman ${ }^{10}$, Nir Barzilai ${ }^{11}$, Dellara F. Terry ${ }^{7}$, Alberto Riva ${ }^{12}$, \\ Chiara Viviani Anselmi ${ }^{3}$, Alberto Malovini ${ }^{3}$, Aya Kitamoto ${ }^{4}$, Motoji Sawabe ${ }^{13}$, Tomio Arai ${ }^{13}$, Yasuyuki \\ Gondo $^{14}$, Martin H. Steinberg ${ }^{6}$, Nobuyoshi Hirose ${ }^{9}$, Gil Atzmon ${ }^{11}$, Gary Ruvkun ${ }^{5}$, Clinton T. Baldwin ${ }^{6 \%}$, \\ Thomas T. Perls ${ }^{70}$
}

1 Department of Biostatistics, Boston University School of Public Health, Boston, Massachusetts, United States of America, 2 Department of Medicine Sections of Infectious Diseases, Boston University School of Medicine, Boston, Massachusetts, United States of America, 3 Department of Genetics, IRCCS Multimedica, Milan, Italy, 4 Computational Systems Biology Research Group, Advanced Science Institute, RIKEN, Yokohama, Kanagawa, Japan, 5 Department of Genetics, Massachusetts General Hospital and Harvard Medical School, Boston, Massachusetts, United States of America, 6 Center for Human Genetics, Boston University School of Medicine, Boston, Massachusetts, United States of America, 7 Geriatrics Section, Department of Medicine, Boston University School of Medicine, Boston, Massachusetts, United States of America, $\mathbf{8}$ Department of Bioengineering, Boston University School of Engineering, Boston, Massachusetts, United States of America, 9 Division of Geriatric Medicine, Department of Internal Medicine, Keio University School of Medicine, Tokyo, Japan, 10 Department of Systems \& Computational Biology, Albert Einstein College of Medicine, Bronx, New York, United States of America, 11 Institute of Aging Research and Diabetes Research Center, Departments of Medicine and Genetics, Albert Einstein College of Medicine, Bronx, New York, United States of America, 12 Department of Molecular Genetics and Microbiology, University of Florida at Gainesville, Florida, United States of America, 13 Department of Pathology, Tokyo Metropolitan Geriatric Hospital, Tokyo, Japan, 14 Clinical Thanatology and Geriatric Behavioral Science, Graduate School of Human Sciences, Osaka University, Suita, Osaka, Japan

\begin{abstract}
Background: The strong familiality of living to extreme ages suggests that human longevity is genetically regulated. The majority of genes found thus far to be associated with longevity primarily function in lipoprotein metabolism and insulin/ IGF-1 signaling. There are likely many more genetic modifiers of human longevity that remain to be discovered.

Methodology/Principal Findings: Here, we first show that 18 single nucleotide polymorphisms (SNPs) in the RNA editing genes $A D A R B 1$ and $A D A R B 2$ are associated with extreme old age in a U.S. based study of centenarians, the New England Centenarian Study. We describe replications of these findings in three independently conducted centenarian studies with different genetic backgrounds (Italian, Ashkenazi Jewish and Japanese) that collectively support an association of ADARB1 and $A D A R B 2$ with longevity. Some SNPs in ADARB2 replicate consistently in the four populations and suggest a strong effect that is independent of the different genetic backgrounds and environments. To evaluate the functional association of these genes with lifespan, we demonstrate that inactivation of their orthologues $a d r-1$ and $a d r-2$ in $C$. elegans reduces median survival by $50 \%$. We further demonstrate that inactivation of the argonaute gene, rde-1, a critical regulator of RNA interference, completely restores lifespan to normal levels in the context of adr-1 and adr-2 loss of function.
\end{abstract}

Conclusions/Significance: Our results suggest that RNA editors may be an important regulator of aging in humans and that, when evaluated in C. elegans, this pathway may interact with the RNA interference machinery to regulate lifespan.

Citation: Sebastiani P, Montano M, Puca A, Solovieff N, Kojima T, et al. (2009) RNA Editing Genes Associated with Extreme Old Age in Humans and with Lifespan in C. elegans. PLoS ONE 4(12): e8210. doi:10.1371/journal.pone.0008210

Editor: Joanna Mary Bridger, Brunel University, United Kingdom

Received July 22, 2009; Accepted November 3, 2009; Published December 14, 2009

Copyright: (C) 2009 Sebastiani et al. This is an open-access article distributed under the terms of the Creative Commons Attribution License, which permits unrestricted use, distribution, and reproduction in any medium, provided the original author and source are credited.

Funding: This work was supported by National Institutes of Health grants: R01 HL087681 (to M.H.S), K24 AG025727 (to T.T.P), R01 AR055115 (to M.M.), RO1 AG027216 (to C.B.), the Italian Ministry of Research and University (to A. P. and A. M), and the following grants to G.A, A.B. and N.B.: AG-027734, AG-18728, RR-12248, DK-20541, and M01-RR12248 and a grant from the Glenn Foundation. M.C.W. is supported by a Life Sciences Research Foundation fellowship. S.E.J.F. is supported by the MGH ECOR Fund for Medical Discovery. The funders had no role in study design, data collection and analysis, decision to publish, or preparation of the manuscript.

Competing Interests: The authors have declared that no competing interests exist.

*E-mail: sebas@bu.edu (PS); mmontano@bu.edu (MM)

9 These authors contributed equally to this work.

I These authors also contributed equally to this work.

\section{Introduction}

Exceptional longevity (EL) in humans, defined as living to extreme old ages such as 100 years and older, is strongly familial [1-8] and the factors that facilitate such exceptional survival have broad public health significance including a marked delay in age-related disability [9-11] and certain age-related diseases [12-14]. Genetically, exceptional longevity is presumed to be a complex trait [15-19]. Several candidate gene association studies have been successful in discovering longevity-associated 
genes in humans. However, these variants have been mainly related to lipoprotein metabolism [20-22], FOXO proteins [23,24], and insulin/IGF-1 signaling [25] It is likely that many more genetic modifiers of human aging have yet to be discovered [25].

In this study, we investigate two genes in the $\mathrm{A}$ (adenosine) to I (inosine) RNA editing pathway, a post-transcriptional process by which adenosine residues are converted to inosine resulting in a change in gene expression or protein function. Targets of RNA editing include a large number of genes as well as micro RNA. Thus, it is not surprising that such a non-specific cellular process would be involved in a general maintenance of cellular health and lifespan. However, such an implication has not been previously demonstrated.

Here, we first report significant association of these genes with EL in four centenarian studies that include the New England Centenarian Study (NECS), with more than 1,500 US individuals of primarily North European ancestry, aged between 90 and 119 years; the Southern Italian Centenarian Study (SICS) - a study of nonagenarians and centenarians from a closed population of Cilento, South Italy; the Ashkenazi Jewish Centenarian Study (AJCS), a study of approximately 300 nonagenarians and centenarians from a founder population of North Eastern European background, all resident in the US; and the Japanese Centenarian Study (JCS), a study of Japanese centenarians that has focused on "semi-supercentenarians" subjects living past 105 years [26]. The characteristics of the four populations allow us to assess the robustness of the associations to varying genetic background and environment.

We further evaluate the functional significance of the RNA editing candidate genes in C. elegans lifespan studies and show that silencing orthologs of these genes reduces median survival by $50 \%$. We also show that life span is fully restored by additional knockdown of an RNA interference gene, supporting the functional role of these genes in determining lifespan and implicating a novel axis for future aging studies.

\section{Results and Discussion}

\section{Selection of Candidate Genes}

We selected the two genes to study for multiple reasons. First, in a preliminary genome wide screening using pooled DNA samples from approximately 130 male centenarians and 130 younger male controls from the NECS [27], we identified several single nucleotide polymorphisms (SNPs) in the RNA-editing genes $A D A R B 1$ (21q22.3), and ADARB2 (10p15.3) that were associated with extreme old age. $A D A R B 1$ exhibited the strongest evidence for genetic association with $5 \mathrm{SNPs}$ that met genome-wide significance, with the posterior odds of allelic association $>1,500$ [27]. The probability of these 5 SNPs simultaneously associated under the null hypothesis of no association was $10^{-13}$ based upon a hyper-geometric distribution. Second, this gene lies in chromosome 2lq21 and trisomy 21 (Down syndrome) resembles accelerated aging, with premature age-related changes including in the skin and hair, increased frequency of premature cataracts, hearing loss, menopause and Alzheimer's disease [28] suggesting that genes in chromosome 21 could affect lifespan. Third, among the top genes identified from the preliminary genetic screen, RNA editing represents a general cellular process that might be expected to improve cellular health; and RNA editing activity has been associated with innate immune response [29,30] and age-related syndromes that include dementia and amyotrophic lateral sclerosis (ALS) [31].

\section{Subjects Selected for the Association Study}

From the NECS, genotype data were obtained from 281 males, aged 96-114 years and 596 females, aged 100-119 years. We selected cutoff ages of 96 years for males and 100 years for females of the NECS to focus on the extreme top $1 \%$ survival based on the U.S. Social Security Administration cohort life table (http://www. ssa.gov/OACT/NOTES/as116/as116LOT.html). NECS referent cohort subjects consisted of 270 spouses of centenarian offspring and children of parents who died at the mean age of 73 years (average life expectancy for the parents' birth cohort). Additional referent subjects were selected from the Illumina iControlDB database using genome-wide genetic matching as detailed in the methods $(n=1635)$. Note that approximately 100 male centenarians included in the pooling-based genome screening overlap with this second set. Given that the overlap is relatively small $(<10 \%)$ and that the subsequent analysis uses a different analytic approach (genotype data from individual subjects), we do not think the overlap is a significant concern.

From the SICS, we used genotype data from 271 males, ages 90-108 years and 188 females, aged 90-109 years (total $=459$ ). Data from 200 male and 132 female SICS referent cohort subjects aged 18-48 years were used in this analysis. From the AJCS, genotype data were obtained from 299 oldest subjects (108 males aged 95 and older and 191 females aged 99 and older) and 269 younger referent cohort subjects (spouses of the offspring of centenarians, aged 85 and younger, without evidence of parental longevity). Four hundred and seventy oldest old subjects (82 males aged 100-110 years and 388 females, aged 100-116 years) and 538 referent cohort subjects (randomly selected Japanese subjects, aged 19-89 years) constituted the Japanese association study. Table 1 reports further summaries of subjects' characteristics. Ages of the extreme old were validated with birth certificates (in the case

Table 1. Study Subjects characteristics.

\begin{tabular}{|c|c|c|c|c|c|c|}
\hline & Males & Females & All & Males & Females & All \\
\hline & \multicolumn{3}{|c|}{ NECS oldest old } & \multicolumn{3}{|c|}{ NECS controls } \\
\hline Sample Size & 281 & 596 & 877 & 149 & 121 & 270 \\
\hline Median Age & 102 & 103 & 103 & 75 & 74 & 75 \\
\hline \multirow[t]{2}{*}{ Age Range } & $96-114$ & $100-119$ & $96-119$ & $58-85$ & 53-85 & $53-85$ \\
\hline & \multicolumn{3}{|c|}{ SICS oldest old } & \multicolumn{3}{|c|}{ SICS controls } \\
\hline Sample Size & 271 & 188 & 459 & 200 & 132 & 332 \\
\hline Median Age & 94 & 98 & 96 & 34 & 32 & 33 \\
\hline \multirow[t]{2}{*}{ Age Range } & 90-109 & 90-109 & 90-109 & $18-48$ & $18-48$ & $18-48$ \\
\hline & \multicolumn{3}{|l|}{ NA } & \multicolumn{3}{|c|}{ Illumina controls } \\
\hline Sample Size & & & & 418 & 1217 & 1635 \\
\hline Median Age & & & & 47 & 46 & 47 \\
\hline \multirow[t]{2}{*}{ Age Range } & & & & $30-75$ & $30-75$ & $30-75$ \\
\hline & \multicolumn{3}{|c|}{ AJCS oldest old } & \multicolumn{3}{|c|}{ AJCS controls } \\
\hline Sample Size & 108 & 191 & 299 & 118 & 151 & 269 \\
\hline Median Age & 99 & 101 & 100 & 77 & 73 & 73 \\
\hline \multirow[t]{2}{*}{ Age Range } & $95-108$ & $99-112$ & $95-112$ & 54-85 & $46-85$ & 46-85 \\
\hline & \multicolumn{3}{|c|}{ JCS oldest old } & \multicolumn{3}{|c|}{ JCS controls } \\
\hline Sample Size & 82 & 388 & 470 & 178 & 360 & 538 \\
\hline Median Age & 104 & 106 & 106 & 21 & 72 & 69 \\
\hline Age Range & $100-111$ & 100-116 & 100-116 & 19-89 & 19-89 & 19-89 \\
\hline
\end{tabular}

Reported are summaries of the last contact ages. doi:10.1371/journal.pone.0008210.t001 
of the JCS, the Basic Resident Registration Card). All subjects were enrolled by studies with Institutional Review Board approval and oversight.

\section{Association of ADARB1 with Exceptional Longevity}

We examined the associations of 31 SNPs in ADARB1 in the NECS and SICS samples using recessive and dominant models with Bayesian logistic regression [32]. The details of the statistical analysis are in the Methods and the significant results are summarized in Table 2 that provides the physical positions and allele frequencies derived from the HapMap for these SNPs, and Table 3 (rows 14-15). Five SNPs in ADARB1 are strongly associated with extreme old age in the NECS, and the association of SNP rs414743 remains significant even after imposing stringent corrections for multiple comparisons (Bayesian significance $<0.05 / 145=0.00035$ where 145 is the overall number of SNPs included in this analysis). The five SNPs tag one region of strong linkage disequilibrium (LD) of the gene (Figure 1). None of the SNPs reached statistical significance in the SICS although the three SNPs rs2838809, rs2838810 and rs2838816 exhibited consistent associations in terms of odds ratios and allele frequencies and, when the NECS and SICS data were combined, the three SNPs remained statistically significant. These three SNPs have extreme minor allele frequencies in the NECS centenarians $(\mathrm{MAF}<0.01)$, while the allele frequencies in the controls are very close to referent allele frequencies from the HapMap (Table 3). Figure 2 displays the scatter plot of genotype intensities generated from BeadStudio that rules out genotyping errors thus suggesting that these are real associations and not artifacts.

To further test the generalizability of these results to other independent groups, we evaluated these associations in the AJCS and the JCS, using a combination of proxy SNPs typed with the Affymetrix platforms and SNPs in Table 3 typed with more traditional techniques (See methods). Table 4 summarizes the results of the replication study of 4 of the 5 SNPs and one additional proxy SNP is in Table 5. None of these SNPs in $A D A R B 1$ replicates the results in the NECS and SICS samples although the significant association of the SNP rs17004734 that is within $2 \mathrm{~Kb}$ from rs414743 is consistent with the presence of longevity associated variants in the region. Because the SNPs used in the NECS and SICS are chosen to best capture the genetic variations of Caucasians from the HapMap, they may not be the correct choice for this founder population and indeed Figure 1 shows a different pattern of LD in ADARB1 in the AJCS subjects. All ADARB1 SNPs in Table 3 and two additional proxy SNPs were genotyped in the JCS subjects and Tables 6 and 7 summarize the results. The last three SNPs in Table 6 show effects that are consistent with the NECS and SICS subjects but do not reach statistical significance, even when the data from the three studies are aggregated. Figure 3 shows the posterior densities of the ORs for the three rare SNPs that are suggestive of association but would need much larger sample sizes to reach statistical significance. The association of two proxy SNPs for rs2838816 in Table 7 is again consistent with the presence of longevity

Table 2. Summary of selected SNPs.

\begin{tabular}{|c|c|c|c|c|c|c|}
\hline Row & SNP & Chr & position & $\begin{array}{l}\text { Risk versus } \\
\text { referent alleles }\end{array}$ & CEPH & JPT \\
\hline 1 & rs10903420 & 10 & 1333726 & $A A \vee A G / G G$ & 0.327 & 0.058 \\
\hline 2 & rs1007147 & 10 & 1341088 & $A A \vee A G / G G$ & 0.312 & 0.091 \\
\hline 3 & rs2805562 & 10 & 1357514 & $A A \vee A G / G G$ & 0.15 & 0.058 \\
\hline 4 & rs884949 & 10 & 1361610 & $A A \vee A C / C C$ & 0.124 & 0 \\
\hline 5 & rs2805533 & 10 & 1374633 & $\mathrm{AA} / \mathrm{AG} \vee \mathrm{GG}$ & 0.77 & 0.92 \\
\hline 6 & rs2387653 & 10 & 1397826 & $A A \vee A G / G G$ & 0.097 & 0 \\
\hline 7 & rs2805535 & 10 & 1450432 & $A A \vee A G / G G$ & 0.699 & 0.151 \\
\hline 8 & rs2805543 & 10 & 1454892 & $A A \vee A G / G G$ & 0.46 & 0.105 \\
\hline 9 & rs3898610 & 10 & 1474759 & $A A \vee A G / G G$ & 0.841 & 0.686 \\
\hline 10 & rs1533484 & 10 & 1481339 & $\mathrm{AA} / \mathrm{AG} \vee \mathrm{GG}$ & 0.442 & 0.791 \\
\hline 11 & rs2676192 & 10 & 1495474 & $A A \vee A G / G G$ & 0.301 & 0.419 \\
\hline 12 & rs2387952 & 10 & 1657365 & $A A \vee A G / G G$ & 0.69 & 0.616 \\
\hline 13 & rs17294019 & 10 & 1659347 & $\mathrm{AA} / \mathrm{AG} \vee \mathrm{GG}$ & 0.196 & 0.012 \\
\hline 14 & rs3788157 & 21 & 45335136 & $A A \vee A G / G G$ & 0.63 & 0.65 \\
\hline 15 & rs414743 & 21 & 45336503 & $A A / A G \vee G G$ & 0.47 & 0.31 \\
\hline 16 & rs2838809 & 21 & 45445866 & $A A \vee A G / G G$ & 0.009 & 0 \\
\hline 17 & rs2838810 & 21 & 45447751 & $A A / A G \vee G G$ & 1 & 1 \\
\hline 18 & rs2838816 & 21 & 45454470 & $A A \vee A G / G G$ & 0.009 & 0 \\
\hline
\end{tabular}

List of 18 SNPs - 13 in the gene ADARB2 (10p15.3) and 5 in the gene ADARB1 (21q22.3) - that are associated with exceptional longevity with either dominant or recessive models. The table reports a sequential number for easy identification in the other tables and figures (column 1 ), the SNP identifier from dbSNP (column 2 ), chromosome and physical position from the human genome NCBI Build 36.3 (columns 3 and 4), the risk versus referent alleles that were associated with exceptional longevity using dominant and recessive models (column 5), the frequencies of the risk allele in the HapMap CEPH and JPT. Note that several of alleles in the Japanese group have allele frequencies that change substantially from the CEPH, and the SNPs in rows 4 and 6 become monomorphic.

doi:10.1371/journal.pone.0008210.t002 


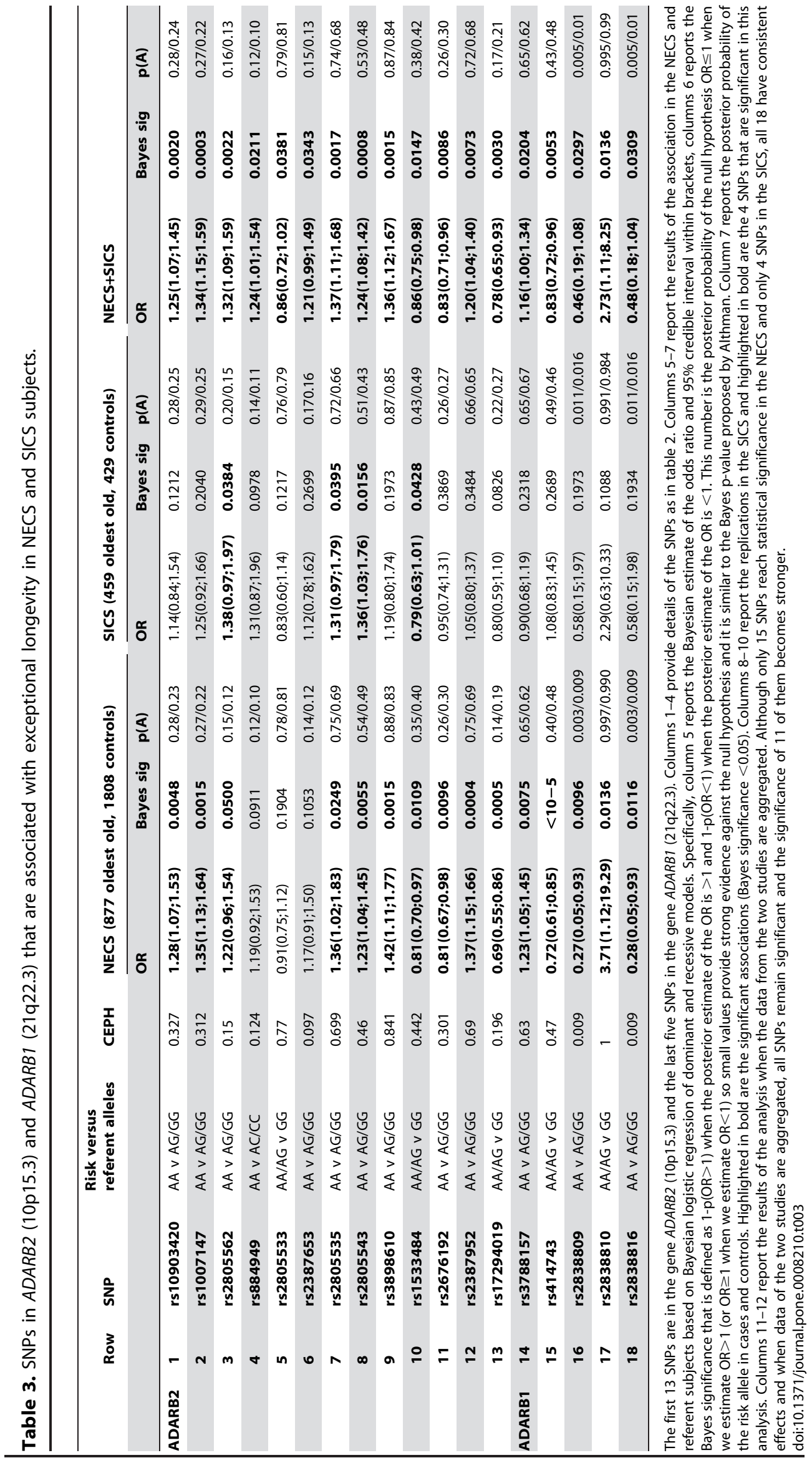



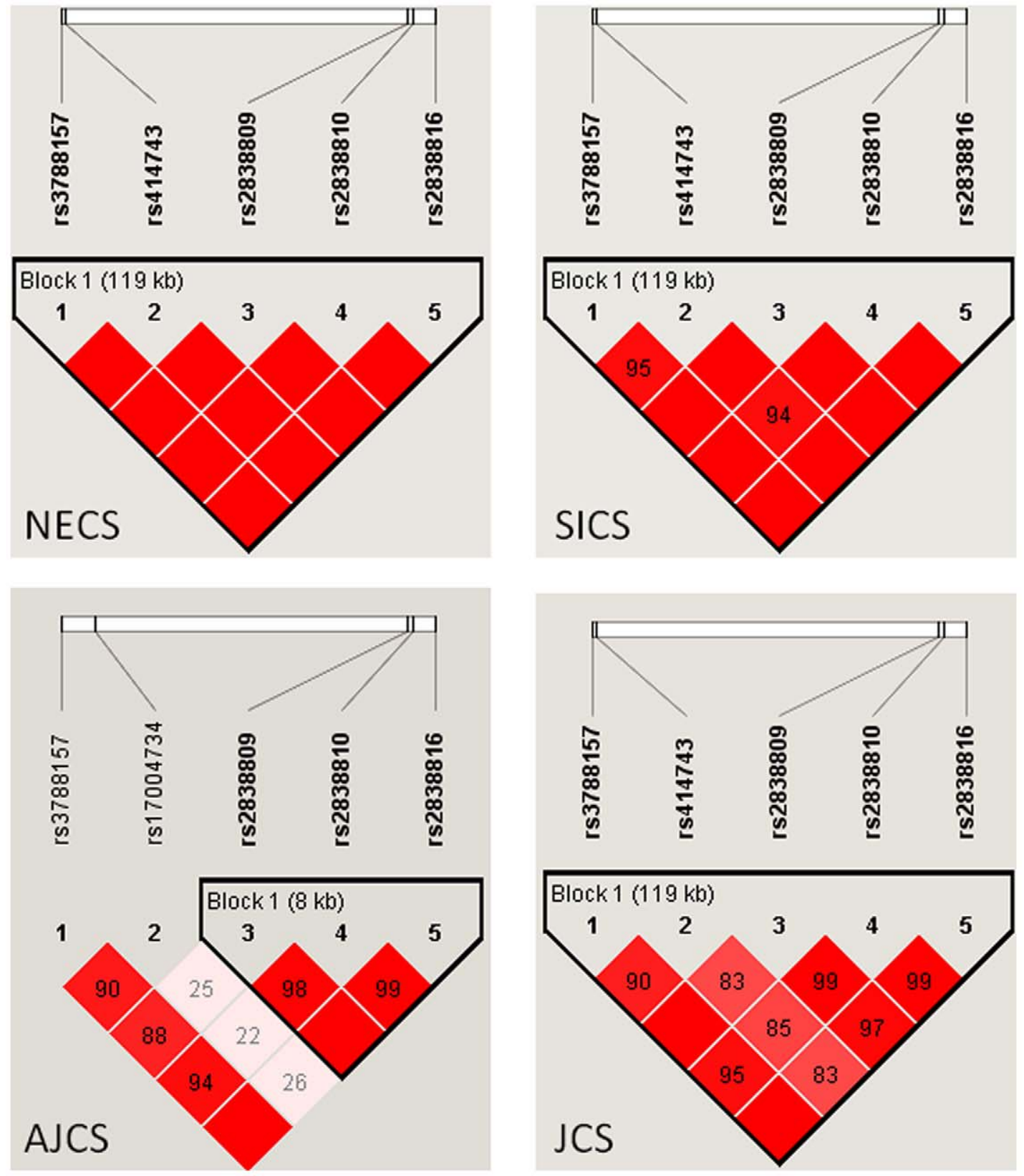

Figure 1. Pattern of LD among the SNP in ADARB1 (chromosome 21) that are associated with exceptional longevity. The four plots display the pattern of LD captured by the SNPs associated with exceptional longevity in ADARB1 (chromosome 21) using data from the NECS, SICS, AJCS and JCS. The intensity of red represents the strength of LD measured by D'.

doi:10.1371/journal.pone.0008210.g001

associated variants in this gene that may not be captured by our SNP selection in the Japanese population.

\section{Association of ADARB2 with Exceptional Longevity}

We examined the associations of 114 SNPs in ADARB2 in the NECS and SICS samples using the same recessive and dominant models. Ten SNPs were strongly associated with extreme old age in the NECS, and one remains significant even after correcting for multiple comparisons (SNPs rs2387952, Bayesian significance $0.0004 \sim 0.05 / 145$ ). Four of these significant SNPs (rs2805562; rs2805533; rs2805543; and rs1533484) were also replicated in the SICS (Bayes significance <0.05) (Table 3). The remaining six SNPs did not reach statistical significance in the SICS but did exhibit consistent associations in terms of odds ratios and allele frequencies and combining data from the NECS and SICS made these ten associations even stronger plus an additional three other SNPs became statistically significant. These SNPs tag a region of approximately $160 \mathrm{~Kb}$ in $A D A R B 2$ that includes two blocks of $\mathrm{LD}$ (Figure 4).

Tables 4 and 5 summarize the results of the replication of 10 of these SNPs in AJCS subjects. Two of the SNPs reach statistical significance in this set (rows 3 and 13) but one has opposite effects compared to the NECS and SICS (rows 13). However, SNPs in rows $1,5-9,11$ and 12 have effects that are consistent with the NECS and SICS and, when the data of the 3 studies are aggregated, they become significant (columns 12-14). The SNPs in 
rs2838809

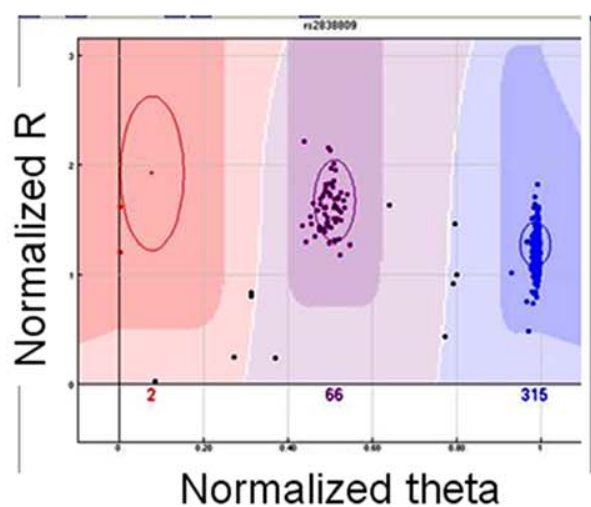

rs2838810

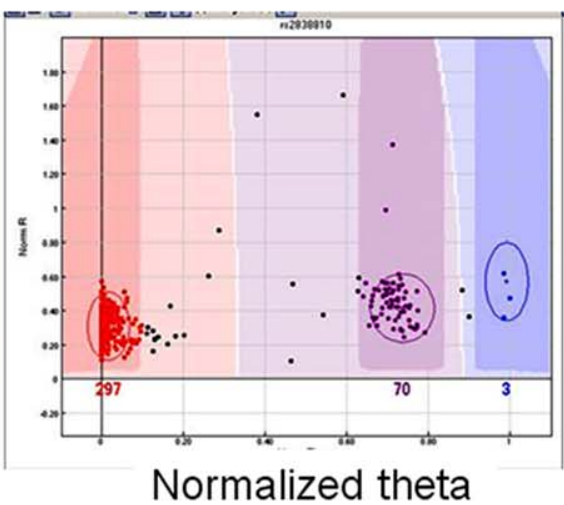

rs2838816

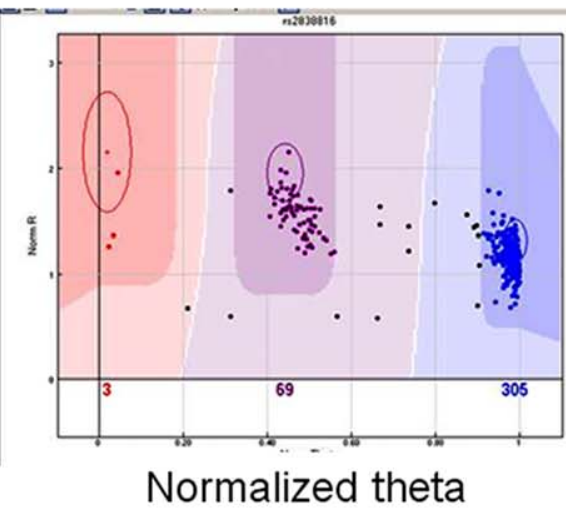

Figure 2. Result of genotype cluster algorithm from BeadStudio. The three plots show the normalized intensities in polar coordinate and the cluster definition from BeadStudio for NECS subjects. The clear separation suggests that the genotype calls are robust. doi:10.1371/journal.pone.0008210.g002

rows 2, 4, and 11 were not typed but proxy SNPs typed with the Affymetrix 6.0 array confirm strong associations of variants in the same region with EL. These SNPs are summarized in Table 5 and are a good proxy for the SNPs originally typed in the NECS and SICS as shown by their proximity in terms of physical distance $<10 \mathrm{~kb}$ and linkage disequilibrium measured by $\mathrm{D}^{\prime}$ and $\mathrm{r}^{2}$.

Only SNP rs2805533 reached statistical significance in the JCS set, with an effect that was consistent with the observed effect in the NECS, SICS and AJCS samples (Table 6). The SNP rs 1533484 was borderline significant (Bayesian significance $\sim 0.06$ ) and demonstrates consistent effects with the NECS and SICS results, but the allele frequencies are substantially different. The SNP rs10903420 was also significant but with an opposite effect compared to the NECS, SICS and AJCS subject sets. Note however the substantial differences in both allele frequencies and pattern of LD that may explain the different patterns of associations in this ethnically very distinct sample. Genotype data of additional SNPs in Table 7 provide further evidence for the existence of variants in the region between physical positions $1340 \mathrm{~K}$ and $1500 \mathrm{~K}$ of chromosome 10 that are associated with exceptional longevity.

\section{Age Trends}

For some SNPs, a clear monotonic pattern associated with increasing age was observed (Figure 5). This monotonic pattern is consistent with a genetic effect that results from alleles positively associated with EL becoming more frequent in older individuals, while alleles that are negatively associated with EL become less frequent. This pattern has also been observed for ApoE alleles [33] and is consistent with the phenomenon of demographic selection [34]. These increases in allele frequencies with age also illustrate the increasing gain of power conferred by studying centenarians and even more so, subjects age 105+ years, in genetic studies of exceptional survival.

\section{Functional Studies}

To go beyond statistical association, we chose to investigate the possible functional role of these genes in regulating lifespan by evaluating their influence on lifespan in the nematode, C. elegans, a robust model organism for candidate lifespan gene discovery. The A-to-I RNA editing gene family and their enzymatic editing activity has been well conserved in a broad array of species including humans, mice, flies, zebrafish, xenopus and notably, $C$. elegans [31,35]. For lifespan analysis, we focused on C. elegans, which has two orthologues of $A D A R B 1$ and $A D A R B 2$ with RNA editing activity, $a d r-1$ and $a d r-2$ [36] (see phylogenetic tree in Figure S1). Because C. elegans has an average lifespan of approximately 20 days, the influence of candidate genes on lifespan can be readily tested. Therefore, to evaluate whether $a d r$ loss-of-function influences C. elegans lifespan, we monitored the lifespan of single and double mutants of $a d r-1(g v 6)$ and $a d r-2(g v 42)$. Both of these alleles are deletions that remove at least a third of the coding sequence and are presumed null alleles [36] (Figure 6a and Figure S2). Strains carrying mutations in $a d r-1$ and $a d r-2$ displayed a shorter lifespan than the wild-type control N2 worms (log-rank test $\left.\mathrm{p}<10^{-8}\right)$. Remarkably, aside from the decline in lifespan, there were no other obvious defects, in contrast with gain-offunction studies that noted lethality in Drosophila [37].

The insulin-like growth factor (IGF) pathway is a well known lifespan regulatory axis in worms [38], flies [39,40], mice [41], and humans [42]. Inactivation of the $C$. elegans insulin like receptor gene daf-2 by dsRNA increased lifespan of both the wild type N2 worms, confirming previous studies. Notably, knockdown of daf-2 by dsRNA also increased lifespan in the background of $a d r-1$ and $a d r-2$ null mutations, resulting in a lifespan phenocopy similar to the wild type N2 worms (Figure 6a). Similar extensions of lifespan were seen with single $a d r-1$ or $a d r-2$ mutants, (Figure S2). These data suggest that IGF axis mediated increases in lifespan due to $d a f-2$ remain active in the presence of $a d r-1$ and $a d r-2$ background genotypes, but with less potency than in a N2 wild type background. From these data, we cannot exclude the possibility that knockdown of RNA editing genes in $a d r-1$ or $a d r-2$ mutants results in increased RNAi activity. In this scenario, increased RNAi might target genes downstream of daf-16 (seven daf-16 gene targets have been identified in comparative analysis of daf- 2 versus daf-2::daf-16 strains [43]), thereby reducing the potency of daf-2 dependent increases in lifespan.

In a previous study, mutations in both $a d r-1$ and $a d r-2$ resulted in increased GFP reporter transgene silencing, suggesting that declines in ADAR function are associated with an increase in RNA interference (RNAi), which would account for the GFP silencing in those experiments. When the argonaute gene $r d e-1$, which is essential for RNA induced silencing complex (RISG) formation, was introduced into $a d r-1 ; a d r-2$ worms containing the transgenes, the increased GFP silencing due to ADAR knockdown was suppressed [44]. This suggests cross-regulation between RNA editors and RNA interference that is further supported by results 


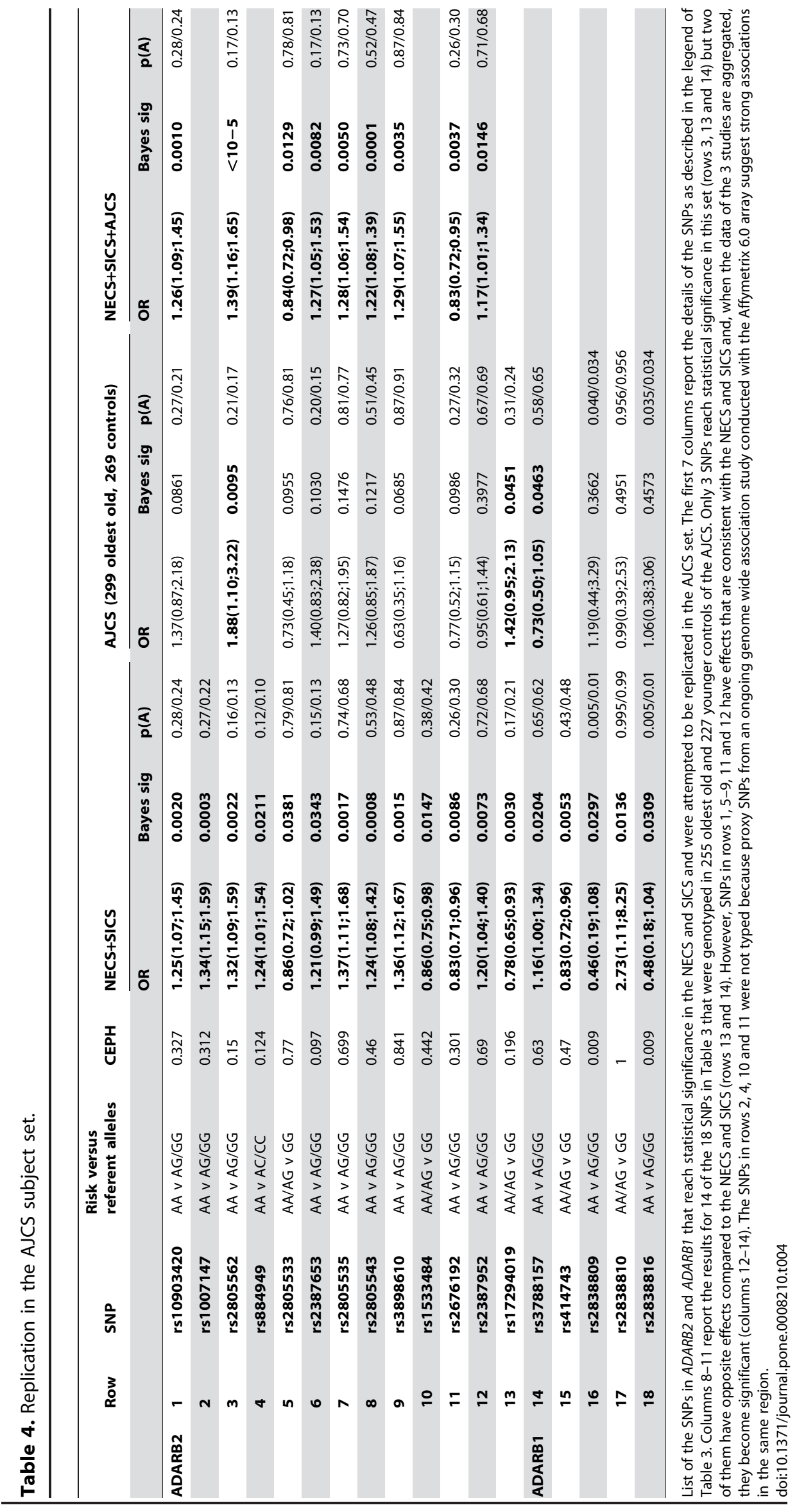




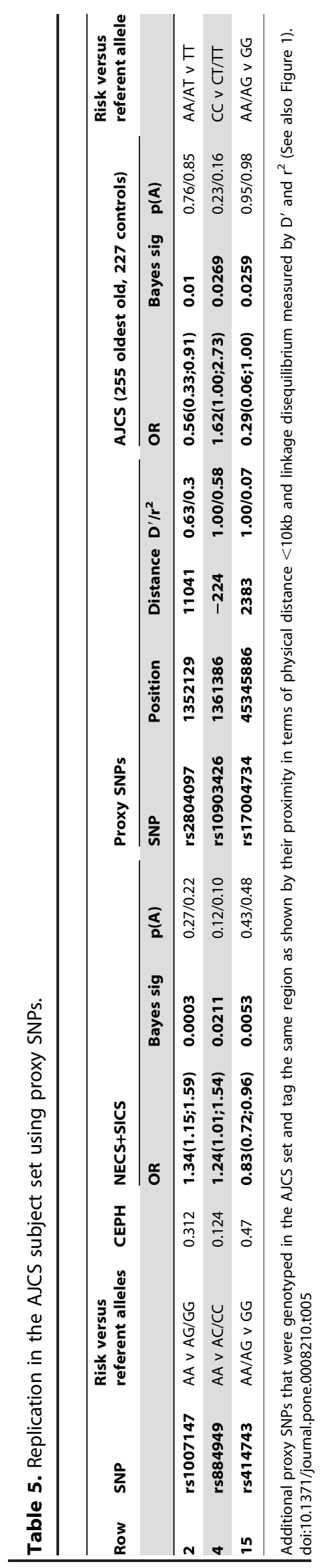

from Tonkin et al [45], wherein they demonstrated that a mild chemotaxis defect of the $a d r-1 ; a d r-2$ double mutant could be rescued by an $r d e-1$ mutant [45]. Therefore, to evaluate the potential for cross-regulation between RNA editing and RNA interference in the context of lifespan, we evaluated $a d r-1 ; a d r-2$ mediated declines in lifespan in the presence of the RNAi defective strain, rde-1(ne-219). Remarkably, and consistent with previous results, the loss of $r d e-1$ completely restored lifespan declines associated with $a d r-1$; $a d r-2$ loss-of-function (Figure 6b). We interpret these data as expanding the interaction between these two RNA regulatory pathways to include lifespan determination.

Our experiments with C. elegans raise the question as to the precise role for A-to-I RNA editing gene activity in human aging, which remains unknown. However, the demonstration that this family of genes is implicated in the regulation of aging in other organisms warrants validation in other species, particularly humans, and provides a novel regulatory axis for future studies on regulatory pathways that influence the aging process. We speculate that as of yet unidentified ADAR variants delay age associated declines in ADAR activity. We note that a reduction in ADAR enzymatic activity is associated with Dementia, ALS and Alzheimers disease in normal aging individuals [46]. Consistent with this interpretation, analysis of published transcriptional profiles of aging in C. elegans indicate that $a d r-1$ and $a d r-2$ expression peak in early adulthood and decline with age rather precipitously. The declines observed in that study are compatible with a protective role for ADAR alleles in aging.

Prior to this study, RNA editing had not been directly implicated in the regulation of aging in humans or C. elegans. Although the impact upon lifespan that we observed in C. elegans appears to be independent of insulin signaling (Figure 6a), the interaction between RNA editing and RNA interference is likely to be complex since decreased insulin signaling in C. elegans can also affect RNA interference [47] and may suggest threshold effects associated with declining levels of insulin signaling. Future studies will be needed to identify and characterize targets of RNA editing and their potential role(s) in modulating RNA interference activity, in the context of aging and age-related diseases.

Our analysis provides strong evidence for association of $A D A R B 1$ and $A D A R B 2$ with extreme old age. Our findings of strongest association in the NECS sample are consistent with that sample being both the largest and oldest of the four studies. The lack of reproducibility for some SNPs may have been due to differences in overall genetic background (ethnicity), size and younger ages of the oldest old samples. Nonetheless, associations were noted across four different study populations suggesting that the associations between $A D A R B 1$ and $A D A R B 2$ and EL are robust to different genetic backgrounds and environmental exposures. $A D A R B 2$ is a very large gene spanning more than $500 \mathrm{~Kb}$ in chromosome 10, but our analysis narrows the association to a region of approximately $100 \mathrm{~Kb}$ that could be followed-up by fine mapping or sequencing for discovering functional variants and to provide a better understanding of the function of these genes in human aging.

\section{Materials and Methods}

\section{Ethic Statement}

Subjects included in the NECS, SICS, AJCS and JCS provided written informed consent, and all research involving human subjects was approved by the Institutional Review Boards of Boston University, Boston, USA (NECS), the "Istituto di Ricovero e Cura a Carattere Scientifico "Multimedica, Milano, Italy (SICS), Albert Eistein College of Medicine, Bronx, USA (AJCS), Keio 
Table 6. Replication in the JCS subject set.

\begin{tabular}{|c|c|c|c|c|c|c|c|c|c|c|c|}
\hline \multirow[b]{3}{*}{ ADARB 2} & \multirow{3}{*}{$\begin{array}{l}\text { Row } \\
1\end{array}$} & \multirow{3}{*}{$\begin{array}{l}\text { SNP } \\
\text { rs10903420 }\end{array}$} & \multirow{3}{*}{$\begin{array}{l}\begin{array}{l}\text { Risk versus } \\
\text { referent alleles }\end{array} \\
A A \vee A G / G G\end{array}$} & \multirow{3}{*}{$\begin{array}{l}\text { CEPH } \\
0.327\end{array}$} & \multicolumn{3}{|l|}{ NECS+SICS } & \multirow{3}{*}{$\begin{array}{l}\text { JPT } \\
0.058\end{array}$} & \multicolumn{3}{|c|}{ JCS (470 oldest old, 538 controls) } \\
\hline & & & & & \multirow{2}{*}{$\begin{array}{l}\text { OR } \\
1.25(1.07 ; 1.45)\end{array}$} & \multicolumn{2}{|c|}{ Bayes sig $p(A)$} & & \multirow{2}{*}{$\begin{array}{l}\text { OR } \\
0.51(0.23 ; 1.07)\end{array}$} & \multicolumn{2}{|c|}{ Bayes sig $p(A)$} \\
\hline & & & & & & 0.0020 & $0.28 / 0.24$ & & & 0.0397 & $0.03 / 0.045$ \\
\hline & 2 & rs1007147 & $A A \vee A G / G G$ & 0.312 & $1.34(1.15 ; 1.59)$ & 0.0003 & $0.27 / 0.22$ & 0.091 & $1.10(0.68 ; 1.81)$ & 0.3435 & $0.08 / 0.075$ \\
\hline & 3 & rs2805562 & $A A \vee A G / G G$ & 0.15 & $1.32(1.09 ; 1.59)$ & 0.0022 & $0.16 / 0.13$ & 0.058 & $0.67(0.26 ; 1.56)$ & 0.1813 & $0.02 / 0.03$ \\
\hline & 4 & rs884949 & $A A \vee A C / C C$ & 0.124 & $1.24(1.01 ; 1.54)$ & 0.0211 & $0.12 / 0.10$ & 0 & & & \\
\hline & 5 & rs2805533 & $A A / A G \vee G G$ & 0.77 & $0.86(0.72 ; 1.02)$ & 0.0381 & $0.79 / 0.81$ & 0.92 & $0.59(0.37 ; 0.92)$ & 0.0110 & $0.88 / 0.93$ \\
\hline & 6 & rs2387653 & $A A \vee A G / G G$ & 0.097 & $1.21(0.99 ; 1.49)$ & 0.0343 & $0.15 / 0.13$ & 0 & & & \\
\hline & 7 & rs2805535 & $A A \vee A G / G G$ & 0.699 & $1.37(1.11 ; 1.68)$ & 0.0017 & $0.74 / 0.68$ & 0.151 & $1.02(0.69 ; 1.49)$ & 0.4690 & $0.14 / 0.14$ \\
\hline & 8 & rs2805543 & $A A \vee A G / G G$ & 0.46 & $1.24(1.08 ; 1.42)$ & 0.0008 & $0.53 / 0.48$ & 0.105 & $0.86(0.57 ; 1.30)$ & 0.2381 & $0.10 / 0.12$ \\
\hline & 9 & rs3898610 & $A A \vee A G / G G$ & 0.841 & $1.36(1.12 ; 1.67)$ & 0.0015 & $0.87 / 0.84$ & 0.686 & $1.09(0.83 ; 1.43)$ & 0.2619 & $0.66 / 0.64$ \\
\hline & 10 & rs1533484 & $A A / A G \vee G G$ & 0.442 & $0.86(0.75 ; 0.98)$ & 0.0147 & $0.38 / 0.42$ & 0.791 & $0.79(0.58 ; 1.07)$ & 0.0641 & $0.76 / 0.80$ \\
\hline & 11 & rs2676192 & $A A \vee A G / G G$ & 0.301 & $0.83(0.71 ; 0.96)$ & 0.0086 & $0.26 / 0.30$ & 0.419 & $0.92(0.71 ; 1.19)$ & 0.2542 & $0.40 / 0.42$ \\
\hline & 12 & rs2387952 & $A A \vee A G / G G$ & 0.69 & $1.20(1.04 ; 1.40)$ & 0.0073 & $0.72 / 0.68$ & 0.616 & $0.98(0.76 ; 1.28)$ & 0.4576 & $0.59 / 0.60$ \\
\hline & 13 & rs17294019 & $\mathrm{AA} / \mathrm{AG} \vee \mathrm{GG}$ & 0.196 & $0.78(0.65 ; 0.93)$ & 0.0030 & $0.17 / 0.21$ & 0.012 & $0.54(0.12 ; 2.03)$ & 0.1846 & $0.01 / 0.02$ \\
\hline \multirow[t]{5}{*}{ ADARB1 } & 14 & rs3788157 & $A A \vee A G / G G$ & 0.63 & $1.16(1.00 ; 1.34)$ & 0.0204 & $0.65 / 0.62$ & 0.65 & $1.03(0.79 ; 1.36)$ & 0.4080 & $0.67 / 0.66$ \\
\hline & 15 & rs414743 & $A A / A G \vee G G$ & 0.47 & $0.83(0.72 ; 0.96)$ & 0.0053 & $0.43 / 0.48$ & 0.31 & $1.14(0.86 ; 1.52)$ & 0.183 & $0.29 / 0.26$ \\
\hline & 16 & rs2838809 & $A A \vee A G / G G$ & 0.009 & $0.46(0.19 ; 1.08)$ & 0.0297 & $0.005 / 0.01$ & 0 & $0.81(0.36 ; 1.81)$ & 0.2959 & $0.028 / 0.034$ \\
\hline & 17 & rs2838810 & $A A / A G \vee G G$ & 1 & $2.73(1.11 ; 8.25)$ & 0.0136 & $0.995 / 0.99$ & 1 & $1.14(0.50 ; 2.60)$ & 0.3791 & $0.974 / 0.970$ \\
\hline & 18 & rs2838816 & $A A \vee A G / G G$ & 0.009 & $0.48(0.18 ; 1.04)$ & 0.0309 & $0.005 / 0.01$ & 0 & $0.81(0.36 ; 1.79)$ & 0.3032 & $0.029 / 0.034$ \\
\hline
\end{tabular}

Lists of the SNPs in ADARB2 and ADARB1 that reach statistical significance in the NECS and SICS and were attempted to be replicated in the JCS set. The first 7 columns report the details of the SNPs as described in the legend of Table 3. Columns 8-11 report the results for 14 of the 18 SNPs in Table 3 that were genotyped in 470 oldest old and 538 younger controls of the JCS. Only 3 SNPs reach statistical significance in this set (rows 1, 5 and 10) but one of them have opposite effects compared to the NECS and SICS (row 1). We did not attempt to merge the results from different populations because of the substantial differences in allele frequencies.

doi:10.1371/journal.pone.0008210.t006

University, Tokyo, Japan (JCS). All data were analyzed anonymously. Control data from the Illumina iControlDB database were anonymized.

\section{SNP Genotyping}

For the NECS and SICS samples, 1 ug of genomic DNA was analyzed on the Illumina 370 CNV chip (Illumina, San Diego, CA) and only samples with at least a $93 \%$ call rate were used for the analysis. For the AJCS and JCS, genotyping was originally performed with the Affymetrix 6.0 chip and 5.0 chips, respectively with required call rates of $99 \%$ or greater. Affymetrix Birdseed algorithm and Illumina Beadstudio were used for genotype calling. Non overlapping SNPs that were not approximated by SNPs with substantial LD $\left(\mathrm{D}^{\prime}>0.8\right)$ were genotyped with Sequenom (AJCS) and BigDye Terminator cycle sequencing kit and an ABI Prism 3730xl DNA analyzer (Applied Biosystems, CA, USA). The sequence data were analyzed with ABI PRISM SeqScape Software version 2.6 (Applied Biosystems).

\section{Creation of a Genetically Matched Control Set}

A referent cohort sample for the NECS subjects was constructed utilizing genotype data from the Illumina iControlDB database and principal components analysis was used to match cases and controls by genetic background. To reduce chances of stratification, we identified 2,077 Caucasian referent subjects from the Illumina iControlDB, all genotype with Illumina arrays, with known age at enrollment between 30 and 75 years, and we used the principal component analysis implemented in the program EIGENSTRAT [48] to examine the structure of this referent group compared to the NECS and SICS subjects. The analysis showed that both the NECS and the Illumina controls are comprised of three major clusters that correspond to northwest, northeast and southwest Europeans, but in the Illumina controls sample there were also subjects with different levels of admixture between the three clusters Figure S3. We therefore randomly sampled 1,538 subjects from the three major clusters to create a control set that matched the genetic background of the NECS extreme old sample set as suggested in [49]. We use the same procedure to identify 81 female and 16 male subjects to be added to the set of SICS controls. The random-selection procedure was repeated twice and lead to the same results.

\section{Genetic Association Analysis (Pooled DNA Samples)}

The statistical analysis of pooling based genome-wide genotype data is described in [27]. Briefly, the method uses Bayesian association tests to score the evidence for allelic associations between centenarians and controls. Prior distributions represent the prior knowledge about the expected number of genes that may be implicated with the trait and therefore correct for multiple comparisons. The analysis also uses linkage disequilibrium (LD) based filters to retain associations that are supported by clusters of SNPs in LD.

\section{Genetic Association Analysis (Individual DNA Samples)}

The genotype data of the $31 \mathrm{SNPs}$ in the genes ADARB1 and 114 SNPs in $A D A R B 2$ were individually analyzed using Bayesian logistic regression [32] to fit dominant and recessive models of inheritance adjusted by gender. The marginal posterior distributions of the ORs were estimated using the implementation of Gibbs sampling in WinBugs 1.4 [50], and the $2.5^{\text {th }}$ and $97.5^{\text {th }}$ 


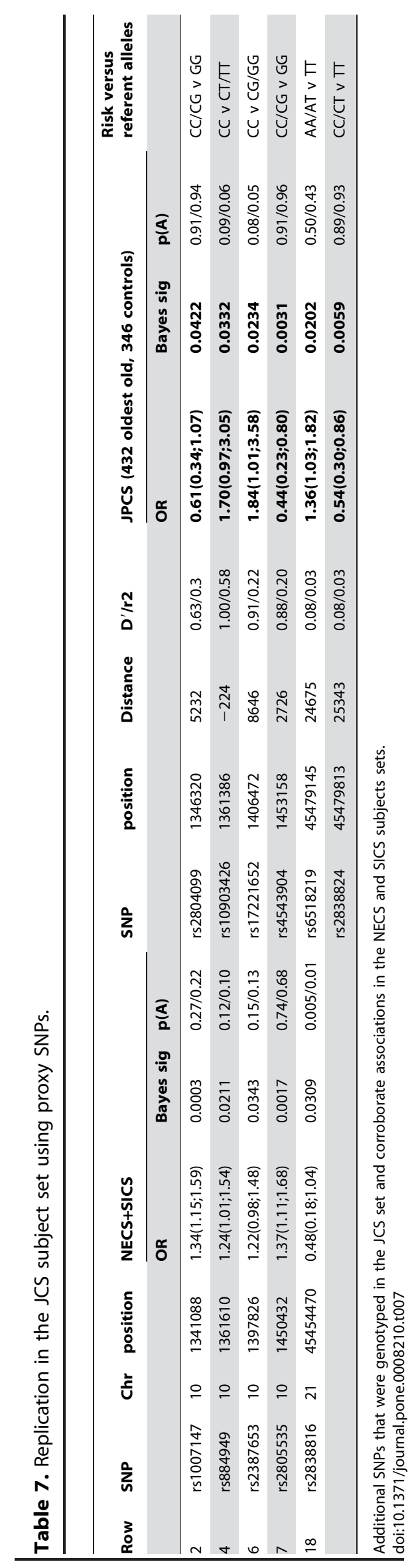

percentile were used to estimate $95 \%$ credible intervals (CI) for the ORs. The $50^{\text {th }}$ percentile was used to estimate the OR, and the frequency of $\mathrm{OR}>1$ was used to estimate the posterior probability $\mathrm{p}(\mathrm{OR}>1)$. The Gibbs sampler was run for at least 10,000 iterations and the last 10,000 simulated values were used to estimate these parameters. We used as prior distributions on the regression coefficients of the logit function normal distributions with mean 0 and standard deviation 3.2 that determine a normal prior distribution of the $\log (\mathrm{OR})$ with mean 0 (no association) and a variance that ranges between 10 with no genetic effect to 40 with a gene $\times$ gender effect. This set of prior distributions was determined to make the analysis robust to rare alleles (frequency<0.10) and we searched for the largest variance that allowed successful execution of the Gibbs sampler. These prior distributions bias the analysis toward the null hypothesis and reduce false positive associations.

The Bayes significance was defined as $1-p(O R>1)$ when the posterior estimate of the $\mathrm{OR}$ was $>1$, and $1-\mathrm{p}(\mathrm{OR}<1)$ when the posterior estimate of the OR was $<1$, and an association was deemed significant in the NECS, SICS, or the data aggregated from the two studies, if the Bayes significance was smaller than 0.05. This measure of significance is the posterior probability of the null hypothesis $\mathrm{OR} \leq 1$ (or $\mathrm{OR} \geq 1$ ) so that small values denotes strong evidence against the null hypothesis [32]. This analysis identified 18 significant SNPs (Table 3), that is more than twice the number expected by chance in 145 independent tests and two SNPs remained significant even after correcting the threshold for the number of tests. Furthermore, the probability that 18 SNPs could be simultaneously found significantly associated under the null hypothesis of no association is 0.0002 , using the binomial distribution with $\mathrm{n}=145, \mathrm{x}=18$ and $\mathrm{p}=0.05$. An association that was significant in the aggregated NECS and SICS data was deemed replicated in either the AJCS or JCS studies if the same SNP was significant (Bayes significance $<0.05$ ) with the same genetic model and consistent effects; or the same SNP did not reach statistical significance (Bayes significance $\geq 0.05$ ), but the ORs in the different studies were in the same direction and when the data from the studies were aggregated, the association was significant. The rationale for the second condition is that both the AJCS and JC have smaller sample sizes, and therefore have less power compared to the NECS. However, consistent effects and increased significance when the aggregated data are analyzed show that the lack of association in the replication study is due to lack of power if effects are similar across different studies. This strategy has been used to increase the power of genetic association studies, see for example [51]. The results are in Tables 4, 5, 6 and 7 .

We conducted a similar analysis stratified by gender but the limited sample sizes did not produce strongly significant results.

\section{Linkage Disequilibrium (LD) Heatmaps}

We used HaploView 4.1 to create the LD heatmaps and LD displays were generated using the $\mathrm{D}^{\prime}$ color scheme where white represents $\mathrm{D}^{\prime}=0$, red represents $\mathrm{D}^{\prime}=1$, and different shades of red represent $0<\mathrm{D}^{\prime}<1$ (Figures 1 and 4 ).

\section{Lifespan Measurements in C. elegans}

To synchronize worms for lifespan, eggs were isolated (N2, adr1, adr-2, adr-1;adr-2, rde-1, rde-4, adr-1;adr-2;rde-1) and synchronized by hatching overnight in the absence of food at 20C. Synchronized L1 larvae were counted and plated (10 worms/plate, $n=60)$ on Escherichia coli bacterial lawns (OP50) on NGM media and allowed to develop to L4-stage larvae at 20C. 5-fluorodeoxyuridine (FudR) solution was added to a final concentration of $0.1 \mathrm{mg} /$ 

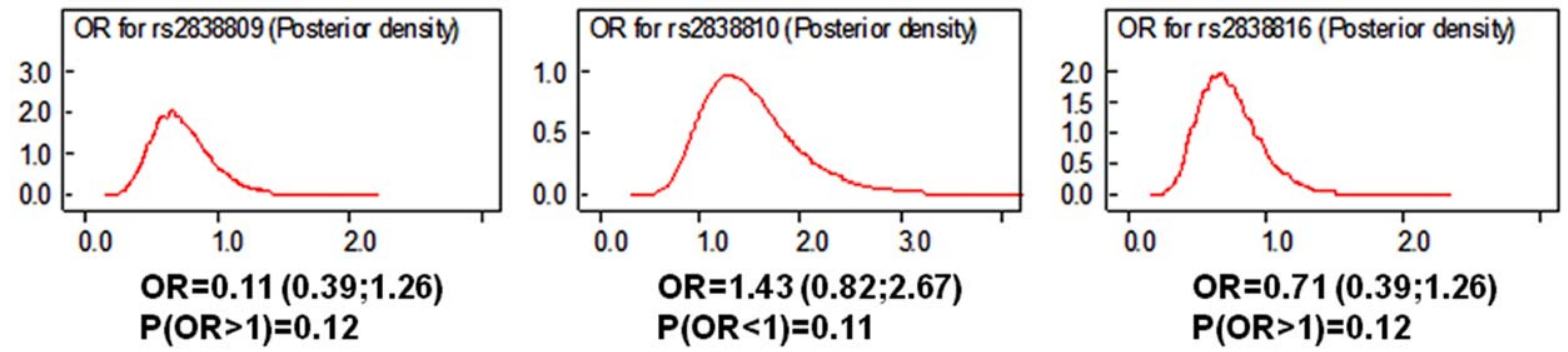

Figure 3. Posterior densities of ORs. Posterior densities of the ORs for the 3 SNPs in ADARB1 with rare alleles and moderate effects in the data aggregated from NECS, SICS and JCS. Significant associations would results in posterior densities not overlapping 1 and definite evidence of either an $\mathrm{OR}<1$ or an $\mathrm{OR}>1$, while all of the three densities have heavy tails and do not provide definite evidence against the null hypothesis of no association. doi:10.1371/journal.pone.0008210.g003

$\mathrm{ml}$ to prevent reproduction. Worms were kept at 20C and lifespan monitored by counting on alternate days. Lifespan was defined as the first day of adulthood (adult lifespan $=0$ ) to death. Aside from reduced lifespan, the worms appeared normal in size and general behavior, consistent with previous reports on $a d r$ mutant strains [36]. We did observe altered chemotaxis during routine passage of the worms to maintain stocks, as previously noted [36]. We also noted reduced progeny viability (data not shown). However, these
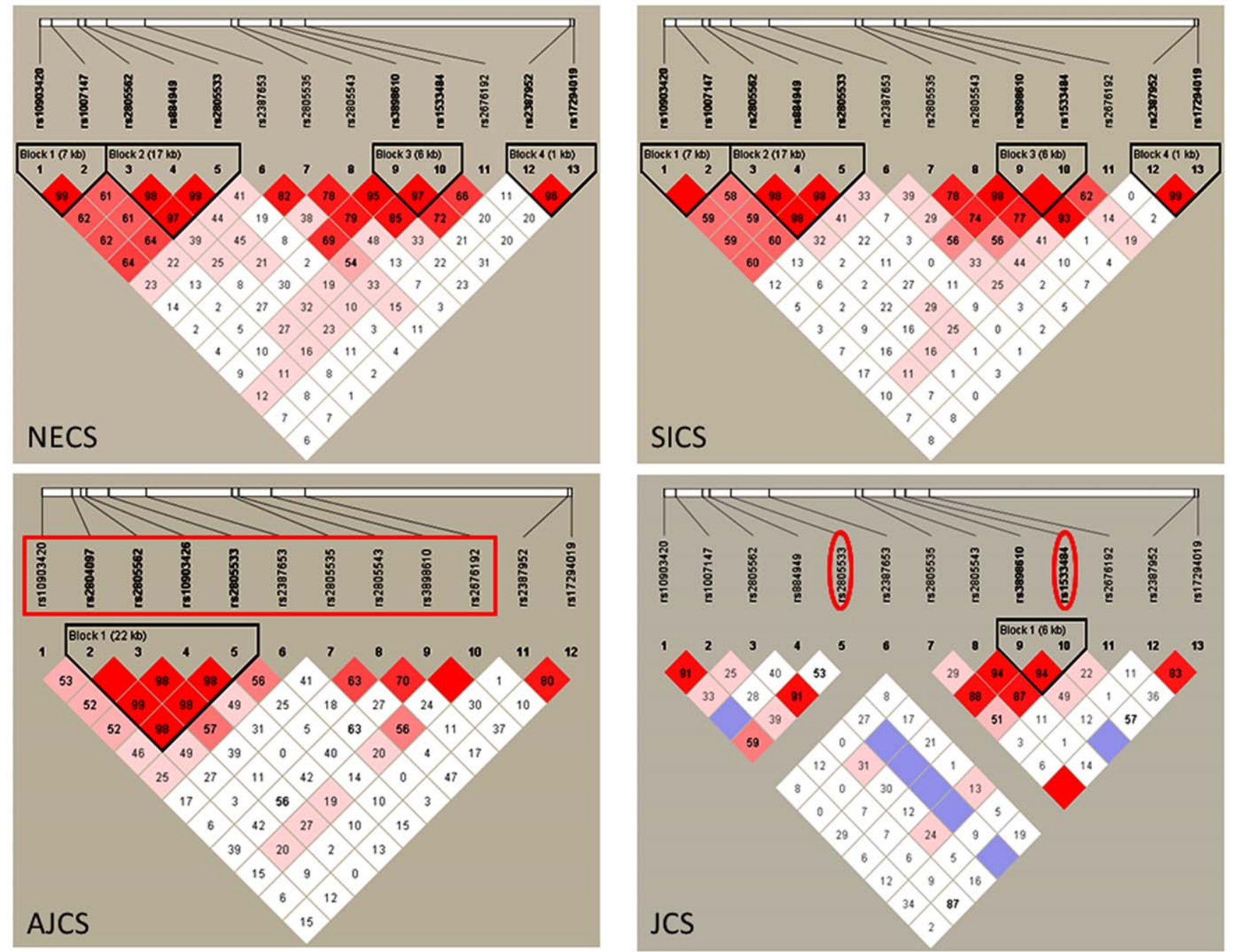

Figure 4. Pattern of LD among the SNP in ADARB2 (chromosome 10) that are associated with exceptional longevity. The four plots display the pattern of LD captured by the SNPs associated with exceptional longevity in ADARB2 (chromosome 10) in the NECS, SICS, AJCS and JCS data. The intensity of red cells represents the strength of LD measured by $D^{\prime}$. The LD pattern in the NECS, SICS and AJCS subjects are very similar but differ substantially from the pattern of LD in the JCS subjects in which two SNPs become almost monomorphic (rs884949 and rs2387653). Highlighted in red are the SNPs that replicate the results in the AJCS and JCS subjects.

doi:10.1371/journal.pone.0008210.g004 

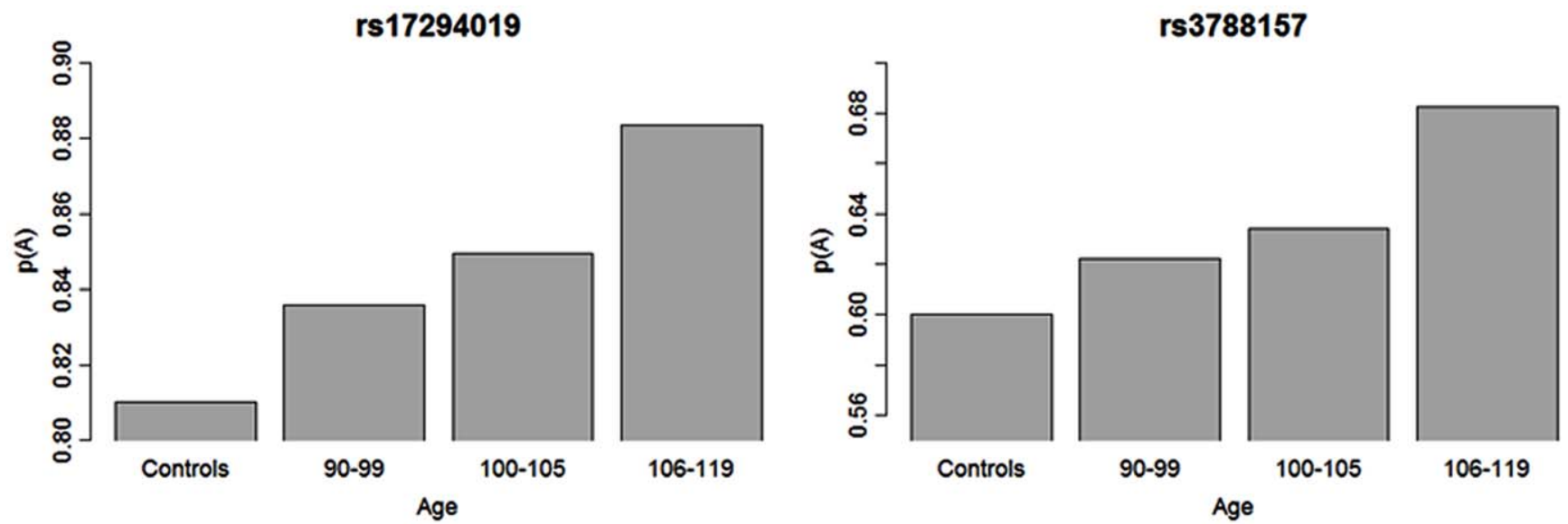

Figure 5. Age related trend of allele frequencies. The two barplots show the age related trend of allele frequencies of SNPs rs 17294019 (ADARB2, SNP \# 98 in Table 1) and rs3788157 (ADARB1, SNP \# 135 in Table 1) in the NECS $(n=1,023)$. The frequencies of the common allele for both SNPs were stratified in the age groups 90-99; 100-105, 106 and higher. Trends of allele frequencies for increasing age groups are consistent with a strong correlation between genotype and phenotype that results in substantial enrichment of protective alleles in older subjects. doi:10.1371/journal.pone.0008210.g005

are unlikely to have influenced our lifespan measurements, since the adult worms were made sterile using FudR and were not transferred during the course of the lifespan assay.
RNAi and Lifespan Measurement

Eggs were isolated from gravid worms and synchronized by hatching overnight in the absence of food. The synchronized Ll
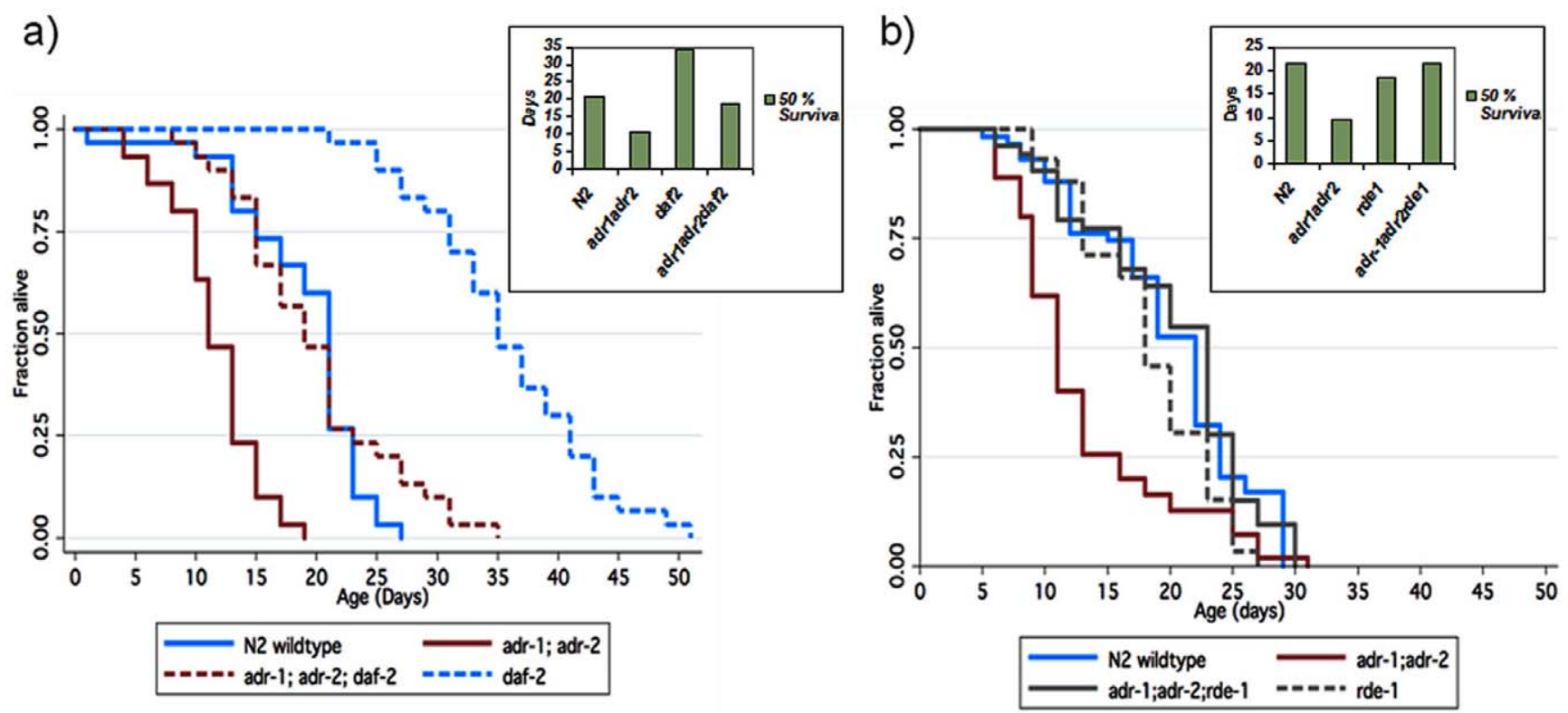

Figure 6. $A D A R$ mediated decline in lifespan, daf-2 influence, and $r d e-1$ rescue. a) Lifespan using mutant strains for adr-1;adr-2 in the context of dsRNA mediated gene inactivation of daf-2. Synchronized worms at the larval stage 4 (L4) were sterilized with FudR and allowed to feed on bacterial lawns that contained dsRNA for daf-2. Note: adr-1; adr-2 double mutant (red solid), adr-1; adr-2 double mutant with dsRNA for daf-2 (red hatched), N2 wild type (blue solid), N2 with dsRNA for daf-2 (blue hatched). Note decline in lifespan due to adr-1; adr-2 compared with N2 wildtype. Also note increases in lifespan of both N2 and adr-1; adr-2 in the presence of dsRNA for daf-2. The 50\% survival time in the adr-1; adr-2 mutant animals was 10 days (95\% limits 9 and 12 days) compared with 20 days (95\% limits 18 and 20 days) for N2 wild-type control worms. RNAi to daf-2 increases lifespan to 34 days ( $95 \%$ limits 32 and 40 days), compared with 20 days for the wild type (N2 worms fed empty vector (RNAi)). daf-2 gene inactivation, in the background of the adr- 1 and adr-2 null mutations also restored lifespan to 18 days ( $95 \%$ limits 16 and 20 days), compared with 10 days for the adr-1;adr-2 double mutant strain. b) Lifespan using mutant strains for adr-1;adr-2 (solid red), N2 wildtype (solid blue), rde-1 (grey hatched), adr-1; adr-2; rde-1 (grey solid) demonstrate declines in lifespan using mutant strains and full rescue of lifespan in an RNAi defective ( $r d e-1)$ background. The adr-1; adr-2 mutant was again about half as long lived as wild-type (median survival time 9 days for adr-1;adr-2 strain (95\% limits 9 and 11 days), and median survival time 21 days (95\% limits 18 and 21 days), for N2 wild-type worms. The survival distribution of the triple mutant adr1; adr-2; rde-1 is median lifespan 21 days (95\% limits 18 and 21 days), which is significantly different from adr-1;adr-2, with a median lifespan of 9 days ( $95 \%$ limits 9 and 11 days). The lifespan of $r d e-1$ was modestly reduced compared with the wild-type N2, as was reported previously ${ }^{29}$. Inset boxes displays 50\% survival (days) for each condition and demonstrates that daf-2 gene inactivation increases lifespan, in both wild type and in adr-1;adr-2 mutant strains (a) and that RNAi knockout (rde-1) restores lifespan.

doi:10.1371/journal.pone.0008210.g006 
larvae were then placed on OP50-containing agar plates and allowed to develop to L4-stage larvae at 20C. The L4-stage larvae were washed thoroughly, and placed either on Escherichia coli HT115 with empty RNAi vector or Escherichia coli HT115 expressing double-stranded RNA (dsRNA) for daf-2. Briefly, dsRNA -expressing bacteria were grown overnight in LB with $50 \mathrm{ug} / \mathrm{ml}$ ampicillin and then seeded onto RNAi NGM plates containing $5 \mathrm{mM}$ isopropylthiogalactoside (IPTG). The RNAi bacteria were induced overnight at room temperature for dsRNA expression. About 30 synchronized L4-stage animals were added to each well and allowed to develop to adults, followed by the addition of FudR. Worms were kept at 20C, and their lifespan was monitored. Worms feeding on bacteria carrying the empty vector were used as a negative control. Log-rank test in the $\mathrm{R}$ package survival was used for the statistical analysis.

\section{Supporting Information}

Figure S1 Phylogenetic clustering and alignment of ADARs form multiple species. Phylogenetic clustering and alignment of ADARs form multiple species, including Human, chimp, bull, cat, rat, chicken, wolf and nematode (C. elegans). Sequences were aligned by using the neighbor-joining algorithm with Clustal X, gap-stripped with corrections for multiple substitutions, and bootstrap analyzed with 1,000 bootstrap resamplings. Phylograms were generated with NJ-plot. The tree is unrooted and was generated using a Neighbor Joining algorithm implemented in Clustal X. Note bootstrap values defining the three main ADAR branches.

Found at: doi:10.1371/journal.pone.0008210.s001 (0.07 MB TIF)

Figure S2 G. elegans lifespan results using individual gene mutant strains. C. elegans lifespan results using individual gene mutant strains, as indicated. Eggs were isolated from gravid worms and synchronized by hatching overnight in the absence of food. The synchronized L1 larvae were then placed on OP50containing agar plates and allowed to develop to L4-stage larvae at 20C. The L4-stage larvae were washed thoroughly, and placed on Escherichia coli expressing double-stranded RNA (dsRNA) for daf-2. Briefly, dsRNA -expressing bacteria were grown overnight in LB with $50 \mathrm{ug} / \mathrm{ml}$ ampicillin and then seeded onto RNAi NGM plates containing $5 \mathrm{mM}$ isopropylthiogalactoside (IPTG). The RNAi daf-2 bacteria were induced overnight at room temperature for daf-2 dsRNA mediated knockdown expression. About 30 synchronized L4-stage animals were added to each well and allowed to develop to adults, followed by the addition of FudR. Worms were kept at 20C, and their lifespan was monitored. Worms feeding on bacteria carrying the empty vector were used as a negative control. Animals were scored every 1 to 2 days subsequently and scored as dead when they no longer responded to gentle prodding.

Found at: doi:10.1371/journal.pone.0008210.s002 (0.05 MB TIF)

\section{References}

1. Christensen K, Johnson TE, Vaupel JW (2006) The quest for genetic determinants of human longevity: challenges and insights. Nat Rev Genet 7: 436-448.

2. Gudmundsson H, Gudbjartsson DF, Frigge M, Gulcher JR, Stefansson K (2000) Inheritance of human longevity in Iceland. Eur J Hum Genet 8: 743-749.

3. Hjelmborg JV, Iachine I, Skytthe A, Vaupel JW, McGue M, et al. (2006) Genetic influence on human lifespan and longevity. Hum Genet 119: 312 321 .

4. Kerber RA, O'Brien E, Smith KR, Cawthon RM (2001) Familial excess longevity in Utah genealogies. J Gerontol A Biol Sci Med Sci 56: B130-139.

5. Perls T, Shea-Drinkwater M, Bowen-Flynn J, Ridge SB, Kang S, et al. (2000) Exceptional familial clustering for extreme longevity in humans. J Am Geriatr Soc 48: 1483-1485.
Figure S3 Population structure of NECS centenarians and controls, SICS centenarians and controls, and Illumina controls. Population structure of NECS centenarians (blue) and controls (red), SICS centenarians (green) and controls (orange), and Illumina controls (grey). Each scatter plot shows the first two principal components that were estimated using genotype data for more than 300K SNPs in NECS, SICS and Illumina subjects using the program Eigenstrat. From top to bottom, left to right: (Blue) scatter plot of the first two principal components in centenarians of the NECS. The two principal components (PGl displayed in the $\mathrm{x}$-axis and PC2 in the y-axis) identify 3 major clusters that based on the ancestry of the NECS centenarians can be labeled as NW Europeans $(\mathrm{PC} 1<0.005$ and $-0.0125<\mathrm{PC} 2<0.0125)$, Ashkenazi Jews (PC1 $>0.005$ and PC2 $<-0.0125)$ and SW Europeans/ Italians $(\mathrm{PC} 1>0.005$ and $\mathrm{PC} 2>0.0125)$. The thresholds on the principal components were identified by splitting the components using mixture models. (Green) scatter plot of the first two principal components in centenarians of the SICS. In agreement with the analysis of NECS subjects, the centenarians of the SICS have a SW European genetic background. (Red) scatter plot of the first two principal components in controls of the NECS that display approximately the same population substructure of centenarians; (Grey) scatter plot of the first two principal components in the Illumina controls. The plots show that the controls have a population substructure similar to the NECS cases and controls but also a larger level of admixture between the three European subgroups. (Orange) scatter plot of the first two principal components in controls of the SICS that exhibit the same SW European genetic background of SICS centenarians. Note that the plot of PC1 and PC2 for NECS controls (red) is repeated twice to facilitate the two comparisons within NECS subjects and between NECS and Illumina controls.

Found at: doi:10.1371/journal.pone.0008210.s003 (0.12 MB TIF)

\section{Acknowledgments}

We are grateful to the subjects and their families of the four centenarian studies. We thank Elixir Pharmaceuticals and Fondazione Longevita for the DNA from referent cohort subjects residing in Cilento, Italy and Illumina for access to its control subject genotype database, and Ms Yukiko Abe, and Ms Sanae Fujimaki (Division of geriatric medicine, Department of Internal medicine, Keio University School of medicine) for their technical assistance and excellent secretarial work.

\section{Author Contributions}

Conceived and designed the experiments: PS MM MHS GR CB TP. Performed the experiments: PS MM TK MCW EM MM SEJF YA CVA AK GA CB. Analyzed the data: PS MM NS SHH AS AM TP. Contributed reagents/materials/analysis tools: PS MM AAP TK SLA SHH YA AB NB DT AR MS TA YG MHS NH GA GR CB TP. Wrote the paper: PS MM NS MHS GR CB TP.

6. Perls TT, Wilmoth J, Levenson R, Drinkwater M, Cohen M, et al. (2002) Lifelong sustained mortality advantage of siblings of centenarians. Proc Natl Acad Sci USA 99: 8442-8447.

7. Schoenmaker M, de Craen AJ, de Meijer PH, Beekman M, Blauw GJ, et al. (2006) Evidence of genetic enrichment for exceptional survival using a family approach: the Leiden Longevity Study. European Journal of Human Genetics 14: 79-84.

8. Terry DF, Wilcox MA, McCormick MA, Pennington JY, Schoenhofen EA, et al. (2004) Lower all-cause, cardiovascular, and cancer mortality in centenarians' offspring. J Am Geriatr Soc 52: 2074-2076.

9. Christensen K, McGue M, Petersen I, Jeune B, Vaupel JW (2008) Exceptional longevity does not result in excessive levels of disability. Proc Natl Acad Sci U S A 105: 13274-13279. 
10. Hitt R, Young-Xu Y, Silver M, Perls T (1999) Centenarians: the older you get, the healthier you have been. Lancet 354: 652 .

11. Terry DF, Sebastiani P, Andersen SL, Perls TT (2008) Disentangling the roles of disability and morbidity in survival to exceptional old age. Arch Intern Med 168: $277-283$.

12. Atzmon G, Schechter G, Greiner W, Davidson D, Rennert G, et al. (2004) Clinical phenotype of families with longevity. J Am Geriatr Soc 52: 274-277.

13. Evert J, Lawler E, Bogan H, Perls T (2003) Morbidity profiles of centenarians: survivors, delayers, and escapers. J Gerontol A Biol Sci Med Sci 58: 232-237.

14. Schoenhofen EA, Wyszynski DF, Andersen S, Pennington J, Young R, et al. (2006) Characteristics of 32 supercentenarians. J Am Geriatr Soc 54: $1237-1240$.

15. Finch CE, Tanzi RE (1997) Genetics of aging. Science 278: 407-411.

16. Salvioli S, Olivieri F, Marchegiani F, Cardelli M, Santoro A, et al. (2006) Genes, ageing and longevity in humans: problems, advantages and perspectives. Free Radic Res 40: 1303-1323.

17. Sorkin J, Post W, Pollin TI, O'Connell JR, Mitchell BD, et al. (2005) Exploring the genetics of longevity in the Old Order Amish. Mech Ageing Dev 126: 347-350.

18. Perls T (2001) Genetic and phenotypic markers among centenarians. J Gerontol A Biol Sci Med Sci 56: M67-70.

19. Perls T, Kunkel L, Puca A (2002) The genetics of aging. Curr Opin Genet Dev 12: 362-369.

20. Barzilai N, Atzmon G, Schechter G, Schaefer EJ, Cupples AL, et al. (2003) Unique lipoprotein phenotype and genotype associated with exceptional longevity. JAMA 290: 2030-2040.

21. Atzmon G, Rincon M, Schechter CB, Shuldiner AR, Lipton RB, et al. (2006) Lipoprotein genotype and conserved pathway for exceptional longevity in humans. PLoS Biol 4: e113.

22. Atzmon G, Pollin TI, Crandall J, Tanner K, Schechter CB, et al. (2008) Adiponectin levels and genotype: a potential regulator of life span in humans. J Gerontol A Biol Sci Med Sci 63: 447-453.

23. Willcox BJ, Donlon TA, He Q Chen R, Grove JS, et al. (2008) FOXO3A genotype is strongly associated with human longevity. Proc Natl Acad Sci U S A 105: 13987-13992.

24. Flachsbart F, Caliebe A, Kleindorp R, Blanche H, von Eller-Eberstein H, et al. (2009) Association of FOXO3A variation with human longevity confirmed in German centenarians. Proc Natl Acad Sci U S A 106: 2700-2705.

25. Vijg J, Campisi J (2008) Puzzles, promises and a cure for ageing. Nature 454: 1065-1071.

26. Kojima T, Kamei H, Aizu T, Arai Y, Takayama M, et al. (2004) Association analysis between longevity in the Japanese population and polymorphic variants of genes involved in insulin and insulin-like growth factor 1 signaling pathways. Exp Gerontol 39: 1595-1598.

27. Sebastiani P, Zhao Z, Abad-Grau MM, Riva A, Hartley SW, et al. (2008) A hierarchical and modular approach to the discovery of robust associations in genome-wide association studies from pooled DNA samples. BMC Genetics 9: 6.

28. Yang Q Rasmussen SA, Friedman JM (2002) Mortality associated with Down's syndrome in the USA from 1983 to 1997: a population-based study. Lancet 359: 1019-1025.

29. Yang JH, Luo X, Nie Y, Su Y, Zhao Q et al. (2003) Widespread inosinecontaining mRNA in lymphocytes regulated by ADAR1 in response to inflammation. Immunology 109: 15-23.

30. Finch CE, Morgan TE (2007) Systemic inflammation, infection, ApoE alleles, and Alzheimer disease: a position paper. Curr Alzheimer Res 4: 185-189.
31. Maas S, Kawahara Y, Tamburro KM, Nishikura K (2006) A-to-I RNA editing and human disease. RNA Biol 3: 1-9.

32. Balding DJ (2006) A tutorial on statistical methods for population association studies. Nat Rev Genet 7: 781-791.

33. Schachter F, Faure-Delanef L, Guenot F, Rouger H, Froguel P, et al. (1994) Genetic associations with human longevity at the APOE and ACE loci. Nat Genet 6: 29-32.

34. Vaupel JW, Carey JR, Christensen K, Johnson TE, Yashin AI, et al. (1998) Biodemographic trajectories of longevity. Science 280: 855-860.

35. Morse DP, Aruscavage PJ, Bass BL (2002) RNA hairpins in noncoding regions of human brain and Caenorhabditis elegans mRNA are edited by adenosine deaminases that act on RNA. Proc Natl Acad Sci U S A 99: 7906-7911.

36. Tonkin LA, Saccomanno L, Morse DP, Brodigan T, Krause M, et al. (2002) RNA editing by ADARs is important for normal behavior in Caenorhabditis elegans. EMBO J 21: 6025-6035.

37. Keegan LP, Brindle J, Gallo A, Leroy A, Reenan RA, et al. (2005) Tuning of RNA editing by ADAR is required in Drosophila. EMBO J 24: 2183-2193.

38. Kenyon C, Chang J, Gensch E, Rudner A, Tabtiang R (1993) A C. elegans mutant that lives twice as long as wild type. Nature 366: 461-464.

39. Clancy DJ, Gems D, Harshman LG, Oldham S, Stocker H, et al. (2001) Extension of life-span by loss of CHICO, a Drosophila insulin receptor substrate protein. Science 292: 104-106.

40. Tatar M, Kopelman A, Epstein D, Tu MP, Yin CM, et al. (2001) A mutant Drosophila insulin receptor homolog that extends life-span and impairs neuroendocrine function. Science 292: 107-110.

41. Holzenberger M, Dupont J, Ducos B, Leneuve P, Geloen A, et al. (2003) IGF-1 receptor regulates lifespan and resistance to oxidative stress in mice. Nature 421: $182-187$.

42. Suh Y, Atzmon G, Cho M-O, Hwang D, Bingrong L, et al. (2008) Functionally significant insulin-like growth factor 1 receptor mutations in centenarians. Proc Natl Acad Sci U S A 105: 3438-3442.

43. Lee SS, Kennedy S, Tolonen AC, Ruvkun G (2003) DAF-16 target genes that control C. elegans life-span and metabolism. Science 300: 644-647.

44. Knight S, Bass B (2002) The role of RNA editing by ADARs in RNAi. Mol Cell Biol 10: 809-817.

45. Tonkin LA, Bass BL (2003) Mutations in RNAi rescue aberrant chemotaxis of ADAR mutants. Science 302: 1725.

46. Lund J, Tedesco P, Duke K, Wang J, Kim SK, et al. (2002) Transcriptional profile of aging in C. elegans. Curr Biol 12: 1566-1573.

47. Wang D, Ruvkun G (2004) The insulin pathway regulates RNAi in C. elegans. Cold Spring Harbor Symposium on Quantitative Biology, 69th edition 69: 429-433.

48. Price AL, Patterson NJ, Plenge RM, Weinblatt ME, Shadick NA, et al. (2006) Principal components analysis corrects for stratification in genome-wide association studies. Nat Genet 38: 904-909.

49. Hinds DA, Stokowski RP, Patil N, Konvicka K, Kershenobich D, et al. (2004) Matching strategies for genetic association studies in structured populations. Am J Hum Genet 74: 317-325.

50. Spiegelhalter DJ, Myles JP, Jones DR, Abrams KR (1999) Methods in health service research. An introduction to bayesian methods in health technology assessment. BMJ 319: 508-512.

51. Thorleifsson G, Walters GB, Gudbjartsson DF, Steinthorsdottir V, Sulem P et al. (2009) Genome-wide association yields new sequence variants at seven loci that associate with measures of obesity. Nat Genet 41: 18-24. 